\title{
TAXONOMIC STUDY ON THE ANGIOSPERMS OF CHAR KUKRI MUKRI WILDLIFE SANCTUARY, BHOLA DISTRICT
}

\author{
MOHAMMAD ZASHIM UDDIN ${ }^{1}$ AND MD. ABIABDULLAH \\ Department of Botany, University of Dhaka, Dhaka-1000, Bangladesh
}

\begin{abstract}
The paper presents the status of angiospermic flora of Char Kukri Mukri Wildlife Sanctuary, a small Island in the Bay of Bengal close to the Char Fassion Upazila of Bhola district. A total of 277 plant species belonging to 76 families was identified from the Island. For each plant species data on scientific name, local name, family, life form and habitat were provided. Trees of this Island were represented by 91 , shrubs by 33 , herbs by 118 and climbers by 35 species. The plant species recorded from the island were distributed in different habitats. Among the habitats, maximum species were recorded in homesteads (104) followed by roadsides (79), mangrove areas (47) and cultivated land (47).The study has reported the presence of medicinal plants, wildlife supporting plants, exotics and invasive plants, rare and threatened plants in the Island. The presence of fruit bearing species in the island is very rare because of high salinity. The introduction of exotics and invasive species into the Island has been recognized as the great challenges to the local angiospermic flora in future. This article also highlights the conservation values, management concerns and some measures for conservation of angiosperm diversity in the Island.
\end{abstract}

Key words: Taxonomic study, Angiosperms, Char Kukri Mukri, Bhola District

\section{Introduction}

Char Kukri Mukri Island is located in the southern side of Char Fassion Upazila of Bhola district is isolated from the main land facing the Bay of Bengal. The total area of the island is about $40 \mathrm{~km}^{2}$. According to local people, human habitation started in the island approximately from 1930 during the British regime. The island was inundated by a big cyclone in 1970 and washed away almost all the people. After the cyclone people again migrated from the main land to the area for fishing and built temporary houses. During the year of 1973/1974, Bangladesh Forest Department started forestation program using the species of Sonneratia apetala (Keora), Avicennia officinalis (Baine) and Excoecaria agallocha (Geoa) in all around the Island. The present planted forest area is about 11307.42 ha (Personal communication with local forest office). Among the forest area, 4973.43 ha is managed by Sadar forest beat and 1360.99 ha by Char Patila beat. The forest is now very dense with many other associated species. Bangladesh Forest Research Institute (BFRI) had introduced plantation trial unit in the island using local mangrove species and some other mesophytic plant species. Such plantation has been performing better in the intertidal zone. The forest bed is muddy and inundated by tidal actions twice

${ }^{1}$ Corresponding author: zashim01@gmail.com 
in a day. The Island is also dissected by 6 small canals and its center part is under huge rice cultivation and human habitation located along the flood protected dams. The soil of the forest is highly alkaline. In 1981, under the Bangladesh Wildlife (Preservation) Amendment Act of 1947, Bangladesh Government has declared the forest area of Char Kukri Mukri as wildlife sanctuary for the protection of its biodiversity. Currently the Island is the dwelling place for 15000 people. The area enjoys a moist tropical maritime climate and rainfall is frequent and heavy during the monsoon season (May to October) ranging between $142 \mathrm{~mm}$ to $1044 \mathrm{~mm}$. Temperature ranges from $16^{\circ} \mathrm{C}$ to $33^{\circ} \mathrm{C}$, whereas humidity ranges from $29 \%$ to $99 \%$ (BBS 2011).

The diversity of plants is very much essential in shaping human civilization in modern days. Unfortunately, such diversity has been eroding in alarming rate from the nature before evaluation and documentation. At the end of 19th century the head of states from all over the world had realized this burning issue. World leaders met in Rieo De Janerio de Janeiro in 1992 to formulate biodiversity conservation policy including agenda 21 which had also given emphasis on the documentation and sustainable utilization of traditional knowledge of plant diversity. After the convention the assessment works of plant diversity in different countries of the world is in progress. In case of Bangladesh angiosperm diversity assessment of different national parks and wildlife sanctuary has already been started (Khan et al. 1994, Rahman and Hassan 1995, Uddin et al. 1998, Uddin and Rahman 1999, Khan and Huq 2001, Uddin et al. 2011, Uddin and Hassan 2004, 2010, Uddin et al. 2013, Uddin et al. 2015 and Sajib et al. 2015). Literature survey revealed that there is no works on the documentation of the angiosperm flora of Char Kukri Mukri wildlife sanctuary. For the management of the sanctuary, baseline data on the flora are essential. In the present study an attempt was made to attain the following objectives: to document the angiosperms, to identify management concerns of the island and to suggest some conservation measures for Char Kukri Mukri wildlife sanctuary.

\section{Materials and Methods}

Extensive floristic survey (Hyland 1972, Balick et al. 1982 and Alexiades 1996) was done in different seasons of the year of 2014 and 2015. The survey included mangrove, cultivated land, roadside and homestead area. Special efforts were given to find species of conservation concern including threatened, endemic and rare species. Sample size was determined using species area curve or species time curve (Goldsmith and Harrison 1976). Maximum identification was done in the field sites and in case of confusion in identification, representative plant specimens were collected and processed using standard herbarium techniques (Hyland 1972). Identification was done by consulting different Floras (Uddin and Hassan 2004, Siddiqui et al. 2007 and Ahmed et al. 2008a, 2008b, 2009a, 2009b, 2009c, 2009d, 2009e). The updated nomenclature of the species are included by following Siddiqui et al. 2007 and Ahmed et al. 2008a, 2008b, 2009a, 2009b, 
2009c, 2009d, 2009e). Threatened categories of plants were confirmed with the help of Khan et al. (2001) and Ara et al. (2013). Some noxious exotic plant species were also identified comparing with the reports of Hossain and Pasha (2004) and Akter and Zuberi (2009). Families were arranged according to Cronquist (1981). Voucher specimens are preserved at Dhaka University Salar Khan Herbarium (DUSH).

\section{Results and Discussion}

A total of 277 plant species belonging to76 families was identified from the Char Kukri Mukri Island. For each plant species scientific name, local name, family, life form and habitat are presented in Table 1. Among the families, Cyperaceae, Poaceae, Fabaceae, Convolvulaceae, Asteraceae, Mimosaceae, Caesalpiniaceae, Euphorbiaceae, Verbenaceae, Amaranthaceae and Acanthanceae were found to be most common. Of 277 species, trees were represented by 91 , shrubs by 33 , herbs by 118 and climbers by 35 species. The plant species recorded from the island were found to be distributed in different habitats. Among the habitats, maximum species were recorded in the homesteads (104) followed by roadsides (79), mangrove areas (47) and cultivated land(47). Most of the plant species in the homesteads and roadsides were introduced by local people, forest department, forest research institute, enthusiastic people and local government. The number of fruit bearing plants was minimum in the island because of high salinity. During survey much attention was paid in the following habitats:

The mangrove plantations were developed all around the island. Each year the newly accreted lands facing the sea were undertaken by forest department under plantation programs. The top canopy in the mangrove was occupied by Sonneratia apetala, Sonneratia caselaoris, Avicennia officinalis, Excoecaria agallocha and Bruguiera gymnorrhiza. Besides few representations of Heritiera fomes, Xylocarpus granatum, Xylocarpus moluccensis, Cerbera mangus, Ceriops decandra, Dolichandronae spathacea, Aegiceros corniculata were also detected in forest. The forest ground was covered mainly by, the seedlings of Excoecaria agallocha, Sonneratia apetala, andAvicennia officinalis. In the forest edge the bush forming dominant species were Acanthus ilicifolius, Dalbergia spinosa, Nipa fruticans, Hibiscus populnea, Thespesia lampus, Sapium indicum and Excoecaria agallocha. The ground near the intertidal zone was mainly dominated by Porteresia coarctata, Zoysia matrella, Cryptocoryne retrospiralis, Zoysia tenuifolia and Saccharum spontaneum. Most common climbers in the mangrove forest were Derris scandens, Derris trifolia, Flagellaria indica, Ipomoea litoralis, and Cercolobus carinatus. Some members of sedge species including Cyperus diformis, Cyperus eragrostis, Cyperus imbricatus and Cyperus lucidus were observed in this zone. The banks of the tidal canals were dominated by a good number of tree species such as Pongamia pinnata, Barringtonia acutangula, Trewia polycarpa, Crataeva nurvala, Heritiera fomes, Nipa fruticans, Tamarindus indica, Sonneratia apetala, Avicennia officinalis, Sonneratia caseolaris, Samanea saman, Albizia procera, Hibiscus populnea, Xylocarpus granatum, Calophylum innophylum, Acacia catechu and Albizia recardiana. 
Table 1. Angiosperms flora of Char Kukri Mukri Island.

\begin{tabular}{|c|c|c|c|c|}
\hline Scientific name & Local name & Family & Habit & Habitat \\
\hline Abelmoschus moschatus Mdedic. & Bonderos & Malvaceae & $\mathrm{H}$ & Roadside \\
\hline Ablemoschus esculentus (L.) Moench & bendi & Malvaceae & $\mathrm{H}$ & Homestead \\
\hline Abutilon indicum (L.) Sweet & - & Malvaceae & $\mathrm{S}$ & Roadside \\
\hline $\begin{array}{l}\text { Acacia auriculiformis A. Cunn. Ex } \\
\text { Benth. \& Hook. }\end{array}$ & Acashmoni & Mimosaceae & $\mathrm{T}$ & Roadside \\
\hline Acacia catechu (L.f.) Willd. & Khiababla & Mimosaceae & $\mathrm{T}$ & Roadside \\
\hline Acacia mangium Willd. & Belgium & Mimosaceae & $\mathrm{T}$ & Roadside \\
\hline Acacia nilotica $\mathrm{L}$. & Babla & Mimosaceae & $\mathrm{T}$ & Homestead \\
\hline Acanthus ilicifolius L. & Hargoza & Acanthaceae & $\mathrm{S}$ & Mangrove \\
\hline Achyranthes aspera $\mathrm{L}$. & Upathlenga & Amaranthaceae & $\mathrm{H}$ & Homestead \\
\hline Adenanthera pavonina $\mathrm{L}$. & Lalchandon & Mimosaceae & $\mathrm{T}$ & Homestead \\
\hline Adhatoda zeylanica Medikus & Bashak & Acanthaceae & $\mathrm{S}$ & Homestead \\
\hline Aegiceras corniculata (L.) Blanco & Khulshi & Myrsinaceae & $\mathrm{S}$ & Mangrove \\
\hline Aegle marmelose (L.) Corr. & Bel & Rutaceae & $\mathrm{T}$ & Homestead \\
\hline Ageratum conyzoides (L.) L. & Fulkuri & Asteraceae & $\mathrm{H}$ & Roadside \\
\hline Albizia lebbeck (L.) Benth. \& Hook. & Shilkoroi & Mimosaceae & $\mathrm{T}$ & Homestead \\
\hline Albizia procera (Roxb.) Benth. & Sadakoroi & Mimosaceae & $\mathrm{T}$ & Homestead \\
\hline $\begin{array}{l}\text { Albizia richardiana (Voigt.) King \& } \\
\text { Prain. }\end{array}$ & Shiris & Mimosaceae & $\mathrm{T}$ & Homestead \\
\hline Albizia saman (Jacq.) Merr. & Botkoroi & Mimosaceae & $\mathrm{T}$ & Homestead \\
\hline Allium tuberosum Rottler ex Spreng. & Chinese leek & Liliaceae & $\mathrm{H}$ & Homestead \\
\hline Alocasia macrorrhizos (L.) G. Don & Mankachu & Araceae & $\mathrm{H}$ & Homestead \\
\hline $\begin{array}{l}\text { Alternanthera philoxeroides (Mart.) } \\
\text { Griseb. }\end{array}$ & Helencha & Amarnthaceae & $\mathrm{H}$ & Cultivated lanc \\
\hline $\begin{array}{l}\text { Alternanthera sessilis (L.) R. Br. Ex } \\
\text { DC.. }\end{array}$ & Hainchashak & Amaranthaceae & $\mathrm{H}$ & Cultivated lanc \\
\hline Amaranthus gangeticus L. & Lashak & Amaranthaceae & $\mathrm{H}$ & Homestead \\
\hline Amaranthus spinosus $\mathrm{L}$. & Kantanote & Amaranthaceae & $\mathrm{H}$ & Roadside \\
\hline Amaranthus viridis $\mathrm{L}$. & Data shak & Amaranthaceae & $\mathrm{H}$ & Homestead \\
\hline Anacardium occidentale L. & Kajubadam & Anacardiaceae & $\mathrm{T}$ & Homestead \\
\hline Annona squamosa $\mathrm{L}$. & Ata & Annonaceae & $\mathrm{T}$ & Homestead \\
\hline $\begin{array}{l}\text { Anodendron paniculatum (Roxb.) A. } \\
\text { DC. }\end{array}$ & - & Asclepiadaceae & $\mathrm{C}$ & Mangrove \\
\hline $\begin{array}{l}\text { Anthocephalus cadamba (Roxb.) } \\
\text { Miq. }\end{array}$ & Kadam & Rubiaceae & $\mathrm{T}$ & Homestead \\
\hline $\begin{array}{l}\text { Aphanamixis polystachya (Wall.) R. } \\
\text { N. Parker }\end{array}$ & Pitraj & Meliaceae & $\mathrm{T}$ & Homestead \\
\hline Aphania danura(Roxb.) Radlk. & Apin & Sapindaceae & $\mathrm{S}$ & Roadside \\
\hline Areca catechu L. & Supari & Arecaceae & $\mathrm{T}$ & Homestead \\
\hline Argyreia argentea (Roxb.) Choisy & - & Convolvulaceae & $\mathrm{C}$ & Roadside \\
\hline Artocarpus heterophyllus Lamk. & Kathal & Moraceae & $\mathrm{T}$ & Roadside \\
\hline Artocarpus lacucha Buch.-Ham. & Dewa & Moraceae & $\mathrm{T}$ & Homestead \\
\hline Averrhoa carambola $\mathrm{L}$. & Kamranga & Averrhoaceae & $\mathrm{T}$ & Homestead \\
\hline Avicennia officinalis L. & Baine & Verbenaceae & $\mathrm{T}$ & Mangrove \\
\hline
\end{tabular}


Contd.

\begin{tabular}{|c|c|c|c|c|}
\hline Scientific name & Local name & Family & Habit & Habitat \\
\hline $\begin{array}{l}\text { Axonopus compressus (Sw.) P. } \\
\text { Beauv. }\end{array}$ & Dhakagass & Poaceae & $\mathrm{H}$ & Roadside \\
\hline Azadirachta indica A. Juss. & Neem & Meliaceae & $\mathrm{T}$ & Homestead \\
\hline Bacopa monnieri (L.) Pennell & Brammi Shak & Scrophulariaceae & $\mathrm{H}$ & Mangrove \\
\hline Bambusa balcooa Roxb. & Baijja Bans & Poaceae & $\mathrm{T}$ & Homestead \\
\hline Barringtonia acutangula (L.) Gaertn. & Hizol & Lecythidaceae & $\mathrm{T}$ & Mangrove \\
\hline Basella rubra $\mathrm{L}$. & Puishak & Basellaceae & $\mathrm{C}$ & Homestead \\
\hline Bauhinia purpurea $\mathrm{L}$. & Kanchan & Caesalpiniaceae & $\mathrm{T}$ & Homestead \\
\hline Benincasa hispida (Thunb.) Cogn. & Chalkumra & Cucurbitaceae & $\mathrm{C}$ & Homestead \\
\hline Blumea lacera (Burm. f.) DC. & Kukurmuta & Asteraceae & $\mathrm{H}$ & Roadside \\
\hline Blumea membranacea Wall.ex DC. & Kukurmuta & Asteraceae & $\mathrm{H}$ & Roadside \\
\hline Bombax ceiba $\mathrm{L}$. & Shimultula & Bombacaceae & $\mathrm{T}$ & Homestead \\
\hline Borassus flabellifer $\mathrm{L}$. & Tal & Arecaceae & $\mathrm{T}$ & Roadside \\
\hline Breynia retusa (Dnnst.) Alston. & - & Euphorbiaceae & $\mathrm{S}$ & Roadside \\
\hline Bruguiera gymnorrhiza (L.) Lamk. & Kakra & Rhizophoraceae & $\mathrm{T}$ & Mangrove \\
\hline Bryophyllum pinnatum (Lamk.)Oken & Pathorkusi & Crassulaceae & $\mathrm{H}$ & Homestead \\
\hline Caesalpinia bunduc (L.) Roxb. & Neta & Caesalpiniaceae & $\mathrm{C}$ & Roadside \\
\hline Caesalpinia crista $\mathrm{L}$. & - & Caesalpiniaceae & $\mathrm{C}$ & Roadside \\
\hline Cajanus cajans (L.) Millsp. & Orhor & Fabaceae & $\mathrm{S}$ & Roadside \\
\hline $\begin{array}{l}\text { Calamus guruba BUch.-Ham. Ex } \\
\text { Martius }\end{array}$ & Bet & Arecaceae & $\mathrm{C}$ & Roadside \\
\hline Calophylum innophylum $\mathrm{L}$. & Kunail & Clusiaceae & $\mathrm{T}$ & Roadside \\
\hline Calotropis gigantea (L.) R. Br. & Akanda & Asclepiadaceae & $\mathrm{S}$ & Roadside \\
\hline Calotropis procera (Aiton) Dryand & Akand & Asclepiadaceae & $\mathrm{S}$ & Roadside \\
\hline Canavalia ensiformis (L.) DC. & Moiseem & Fabaceae & $\mathrm{C}$ & Roadside \\
\hline Canavalia maritime Thou. & - & Fabaceae & $\mathrm{C}$ & Roadside \\
\hline Capsicum frutescens $\mathrm{L}$. & Morich & Solanaceae & $\mathrm{H}$ & Cultivated lanc \\
\hline Carex caricinus $\mathbf{L}$. & Sedge & Cyperaceae & $\mathrm{H}$ & Mangrove \\
\hline Carica papaya $\mathrm{L}$. & Pepe & Caricaceae & $\mathrm{S}$ & Homestead \\
\hline Cassia alata $\mathrm{L}$. & Dadmordan & Caesalpiniaceae & $\mathrm{S}$ & Homestead \\
\hline Cassia fistula $\mathrm{L}$. & Sonalu & Caesalpiniaceae & $\mathrm{T}$ & Roadside \\
\hline Cassia occidentalis $\mathrm{L}$. & - & Caesalpiniaceae & $\mathrm{H}$ & Roadside \\
\hline Cassia siamea Lamk. & Minjori & Caesalpiniaceae & $\mathrm{T}$ & Roadside \\
\hline Cassia tora $\mathrm{L}$. & - & Caesalpiniaceae & $\mathrm{H}$ & Roadside \\
\hline Casuarina equisetifolia $\mathrm{L}$. & Jau & Casuarinaceae & $\mathrm{T}$ & Roadside \\
\hline Cayratia japonica (Thunb.) Gagnep. & - & Vitaceae & $\mathrm{C}$ & Roadside \\
\hline Celosia cristata $\mathrm{L}$. & Morogful & Amaranthaceae & $\mathrm{H}$ & Homestead \\
\hline Centella asiatica (L.) Urban & Adamoni & Apiaceae & $\mathrm{H}$ & Roadside \\
\hline Cerbera manghas $\mathrm{L}$. & Cerbera & Apocynaceae & $\mathrm{T}$ & Mangrove \\
\hline Ceriops decandra (Griff.) Ding. Hou & Goran & Rhizophoraceae & $\mathrm{T}$ & Mangrove \\
\hline $\begin{array}{l}\text { Chrysalidocarpus lutescens (Bory) } \\
\text { H. Wendl. }\end{array}$ & Arecapalm & Arecaceae & $\mathrm{T}$ & Homestead \\
\hline Chrysopogon aciculatus (Retz.) Trin. & Premkanta & Poaceae & $\mathrm{H}$ & Roadside \\
\hline $\begin{array}{l}\text { Citrus aurantifolia } \\
\text { (Christm.\&Panzer) Swingle }\end{array}$ & Lebu & Rutaceae & $\mathrm{S}$ & Homestead \\
\hline
\end{tabular}


Contd.

\begin{tabular}{|c|c|c|c|c|}
\hline Scientific name & Local name & Family & Habit & Habitat \\
\hline Citrus maxima (Burm. F.) Merr. & Jambura & Rutaceae & $\mathrm{T}$ & Homestead \\
\hline Clerodendrum indicum (L.) Kuntze & Bhat & Verbenaceae & $\mathrm{S}$ & Mangrove \\
\hline Clerodendrum inerme (L.) Gaertn. & - & Verbenaceae & $\mathrm{S}$ & Magrove \\
\hline Clerodendrum Viscosum Vent. & Bhat & Verbenaceae & $\mathrm{H}$ & Mangrove \\
\hline Clitorea turnetea $\mathrm{L}$. & Aparajita & Fabaceae & $\mathrm{C}$ & Homestead \\
\hline Cocos nucifera $\mathrm{L}$. & Narikel & Arecaceae & $\mathrm{T}$ & Homestead \\
\hline Codiaeum variegatum (L.) A. Juss. & Patabahar & Euphorbiaceae & $\mathrm{S}$ & Homestead \\
\hline Coix lacryma-jobi $\mathrm{L}$. & - & Poaceae & $\mathrm{H}$ & Roadside \\
\hline Colocasia esculenta (L.) Schott & Kachu & Araceae & $\mathrm{H}$ & Homestead \\
\hline $\begin{array}{l}\text { Cotula hemispherica (Roxb.) Wall.ex } \\
\text { CB. Clarke }\end{array}$ & Cotula & Asteraceae & $\mathrm{H}$ & Cultivated land \\
\hline Crateva nurvala Buch.-Ham. & Borun & Capparadiaceae & $\mathrm{T}$ & Mangrove \\
\hline Crinum amoenum Roxb. & Bonroshun & Liliaceae & $\mathrm{H}$ & Mangrove \\
\hline Crinum asiaticum $\mathrm{L}$. & Crinum & Liliaceae & $\mathrm{H}$ & Mangrove \\
\hline Crotalaria juncea $\mathrm{L}$. & Junjuni & Fabaceae & $\mathrm{H}$ & Roadside \\
\hline Croton bonplandianus Baill. & Bankhira & Euphorbiaceae & $\mathrm{H}$ & Roadside \\
\hline $\begin{array}{l}\text { Chrozophora plicata (Vahl.) A. Juss. } \\
\text { ex. Spreng. }\end{array}$ & - & Euphorbiaceae & $\mathrm{H}$ & Roadside \\
\hline $\begin{array}{l}\text { Cryptocoryne retrospiralis (Roxb.) } \\
\text { Fisch. }\end{array}$ & Kelakachu & Araceae & $\mathrm{H}$ & Mangrove \\
\hline Cucurbita maxima Duchesne & Misti kumra & Cucurbitaceae & $\mathrm{C}$ & Homestead \\
\hline Curcuma domestica Valet. & Halud & Zingiveraceae & $\mathrm{H}$ & Homestead \\
\hline Curcuma gedoaria (Christm.) Rosc. & Shadi & Zingiveraceae & $\mathrm{H}$ & Roadside \\
\hline Cuscuta reflexa Roxb. & Shwarnalata & Cuscutaceae & $\mathrm{C}$ & Roadside \\
\hline Cyclea barbata Miers. & Patalpur & Menispermaceae & $\mathrm{C}$ & Roadside \\
\hline Cynodon dactylon (L.) Pers. & Durbagass & Poaceae & $\mathrm{H}$ & Homestead \\
\hline Cynometra ramiflora $\mathrm{L}$. & Singra & Fabaceae & $\mathrm{T}$ & Mangrove \\
\hline Cyperus diformis $\mathrm{L}$. & Sedge & Cyperaceae & $\mathrm{H}$ & Mangrove \\
\hline Cyperus eragrostis Vahl. & Sedge & Cyperaceae & $\mathrm{H}$ & Mangrove \\
\hline Cyperus imbricatus Retz. & Sedge & Cyperaceae & $\mathrm{H}$ & Mangrove \\
\hline Cyperus lucidus & Sedge & Cyperaceae & $\mathrm{H}$ & Mangrove \\
\hline Cyperus rotundus $\mathrm{L}$. & Muthagass & Cyperaceae & $\mathrm{H}$ & Cultivated land \\
\hline Dalbergia sissoo DC. & Shissu & Fabaceae & $\mathrm{T}$ & Roadside \\
\hline Dalbergia spinosa Roxb. & Tamu & Fabaceae & $\mathrm{S}$ & Mangrove \\
\hline Delonix regia Rafin. & Krishnachura & Caesalpiniaceae & $\mathrm{T}$ & Roadside \\
\hline Dentella repens (L.) J. R. \& G. Forst. & Bhuipat & Rubiaceae & $\mathrm{H}$ & Cultivated land \\
\hline Derris scandens (Roxb.) Benth. & Kalilata & Fabaceae & $\mathrm{C}$ & Mangrove \\
\hline Derris trifoliata Lour. & Kalilota & Fabaceae & $\mathrm{C}$ & Mangrove \\
\hline Dillenia indica $\mathrm{L}$. & Chalta & Dilleniaceae & $\mathrm{T}$ & Homestead \\
\hline Dioscorea alata $\mathrm{L}$. & Jora alu & Dioscoriaceae & $\mathrm{C}$ & Homestead \\
\hline Dioscorea bulbifera $\mathrm{L}$. & Matialu & Dioscoriaceae & $\mathrm{C}$ & Homestead \\
\hline Diospyros blancoi A. DC. & Bilatigab & Ebenaceae & $\mathrm{T}$ & Homestead \\
\hline Diospyros malabarica (Desr.) Kostel. & Deshigab & Ebenaceae & $\mathrm{T}$ & Homestead \\
\hline $\begin{array}{l}\text { Dolichandrone spathacea (L.f.) K. } \\
\text { Schum. }\end{array}$ & Chamhechandan & Bignoniaceae & $\mathrm{T}$ & Mangrove \\
\hline
\end{tabular}


Contd.

\begin{tabular}{|c|c|c|c|c|}
\hline Scientific name & Local name & Family & Habit & Habitat \\
\hline Eclipta prostrata (L.) Mant. & Keshoraj & Asteraceae & $\mathrm{H}$ & Cultivated land \\
\hline Ehretia serrata Roxb. & & Boraginaceae & $\mathrm{T}$ & Roadside \\
\hline Eichhornia crassipes (Mart.) Solms & Kachripana & Pontederiaceae & $\mathrm{H}$ & Homestead \\
\hline Elaeocarpus tectorius (Lour.) Poir. & Jolpai & Elaeocarpaceae & $\mathrm{T}$ & Cultivated land \\
\hline $\begin{array}{l}\text { Eleocharis geniculata (L.) Roem. \& } \\
\text { Schult. }\end{array}$ & Joraghasi & Cyperaceae & $\mathrm{H}$ & Cultivated land \\
\hline Eleusine indica (L.) Gaertn. & Malankuri & Poaceae & $\mathrm{H}$ & Cultivated land \\
\hline Eryngium foetidum $\mathrm{L}$. & Shamadoine & Apiaceae & $\mathrm{H}$ & Homestead \\
\hline Erythrina indica Lamk. & Painnamandar & Fabaceae & $\mathrm{T}$ & Homestead \\
\hline Erythrina ovalifolia Roxb. & Mandar & Fabaceae & $\mathrm{T}$ & Homestead \\
\hline Eucalyptus camaldulensis Dehnhardt & Eucalyptus & Myrtaceae & $\mathrm{T}$ & Roadside \\
\hline Excoecaria agallocha $\mathrm{L}$. & Geoa & Euphorbiaceae & $\mathrm{T}$ & Mangrove \\
\hline Ficus benghalensis L. & Bot & Moraceae & $\mathrm{T}$ & Roadside \\
\hline Ficus hispida L. f. & Dumur & Moraceae & $\mathrm{T}$ & Homestead \\
\hline Ficus infectoria Roxb. & Pakur & Moraceae & $\mathrm{T}$ & Roadside \\
\hline Ficus racemosa $\mathrm{L}$. & Jogdumur & Moraceae & $\mathrm{T}$ & Roadside \\
\hline Ficus rumphii Blume. & Pakur & Moraceae & $\mathrm{T}$ & Roadside \\
\hline Fimbristylis acuminata Vahl & - & Cyperaceae & $\mathrm{H}$ & Cultivated land \\
\hline Fimbristylis dichotoma (L.) Vahl & Fimbristylis & Cyperaceae & $\mathrm{H}$ & Roadside \\
\hline Fimbristylis ferruginea (L.) Vahl & - & Cyperaceae & $\mathrm{H}$ & Cultivated land \\
\hline Flcourtia indica (Burm.f.) Merr. & Paniala & Flacourtiaceae & $\mathrm{T}$ & Homestead \\
\hline Flumeria alba $\mathrm{L}$. & Katgolap & Combretaceae & $\mathrm{T}$ & Homestead \\
\hline Flagellaria indica $\mathrm{L}$. & & Flagellariaceae & $\mathrm{C}$ & Mangrove \\
\hline Garcinia cowa Roxb. ex DC. & Kao & Clusiaceae & $\mathrm{T}$ & Homestead \\
\hline Gardenia jasminoides J.Ellis & Gandhraj & Rubiaceae & $\mathrm{S}$ & Homestead \\
\hline Gmelina arborea Roxb. & Gamari & Verbenaceae & $\mathrm{T}$ & Roadside \\
\hline Gomphrena globosa $\mathrm{L}$. & Botamphul & Amaranthaceae & $\mathrm{H}$ & Roadside \\
\hline Gosypium herbaceum L. & Karpustula & Malvaceae & $\mathrm{H}$ & Roadside \\
\hline Grangea maderaspatana (L.) Poir. & Nemuti & Asteraceae & $\mathrm{H}$ & Cultivated land \\
\hline Heliotropium curassavicum L. & Nuinna & Boraginaceae & $\mathrm{H}$ & Cultivated land \\
\hline Heliotropium indicum $\mathrm{L}$. & Hatisur & Boraginaceae & $\mathrm{H}$ & Cultivated land \\
\hline Heritiera fomes Buch.-Ham. & Sundari & Sterculiaceae & $\mathrm{T}$ & Mangrove \\
\hline Hibiscus rosa-sinensis $\mathrm{L}$. & Joba & Malvaceae & S & Homestead \\
\hline Hydrilla verticilata (L.f.) Royle & Jaji & Hydrocaritaceae & $\mathrm{H}$ & Cultivated land \\
\hline Hygrophila phlomoides Nees & - & Acanthaceae & $\mathrm{H}$ & Cultivated land \\
\hline Hygrophila salicifolia (Vahl) Nees & Kakmasha & Acanthaceae & $\mathrm{H}$ & Cultivated land \\
\hline Imperata cylendrica (L.) P.Beauv. & Ulu & Poaceae & $\mathrm{H}$ & Roadside \\
\hline Ipomea batata (L.) Lamk. & Mistialu & Convolvulaceae & $\mathrm{C}$ & Homestead \\
\hline Ipomoea aquatica Forssk. & Kolmi & Convolvulaceae & $\mathrm{H}$ & Homestead \\
\hline Ipomoea fistulosa Mart. ex Choisy & Dolkolmi & Convolvulaceae & $\mathrm{H}$ & Roadside \\
\hline Ipomoea littoralis Blume. & - & Convolvulaceae & $\mathrm{C}$ & Mangrove \\
\hline Ipomoea pes-caprae (L.) R. Br. & Chagalkhuri & Convolvulaceae & $\mathrm{H}$ & Cultivated land \\
\hline Justicia gendarussa Burm.f. & Justicia & Acanthaceae & $\mathrm{H}$ & Roadside \\
\hline Kyllinga sesquiflora Torr. & Sedge & Cyperaceae & $\mathrm{H}$ & Cultivated land \\
\hline
\end{tabular}


Contd.

\begin{tabular}{|c|c|c|c|c|}
\hline Scientific name & Local name & Family & Habit & Habitat \\
\hline $\begin{array}{l}\text { Kyllinga nemoralis (J.R.Forst. \& G. } \\
\text { Forst) Dandy ex Hutchins\&Dalziel }\end{array}$ & Sedge & Cyperaceae & $\mathrm{H}$ & Cultivated land \\
\hline Lablab purpurea (L.) Sweet & Seem & Fabaceae & $\mathrm{C}$ & Homestead \\
\hline Lagenaria siceraria (Molina) Standl. & Lao & Cucurbitaceae & $\mathrm{C}$ & Homestead \\
\hline Lagerstroemia indica $\mathrm{L}$. & Cheri & Lythraceae & $\mathrm{T}$ & Homestead \\
\hline Lagerstroemia speciosa (L.) Pers. & Jarul & Lythraceae & $\mathrm{T}$ & Homestead \\
\hline $\begin{array}{l}\text { Lannea coromandelica (Houtt.) } \\
\text { Merr. }\end{array}$ & Bhadi & Anacardiaceae & $\mathrm{T}$ & Homestead \\
\hline Lathyrus sativus L. & Khesari & Fabaceae & $\mathrm{H}$ & Cultivated land \\
\hline Lawsonia inermis $\mathrm{L}$. & Mehendi & Lythraceae & $\mathrm{T}$ & Homestead \\
\hline $\begin{array}{l}\text { Leucaena leucocephala (Lamk.) de } \\
\text { Wit. }\end{array}$ & Epilepil & Mimosaceae & $\mathrm{T}$ & Roadside \\
\hline Lindernia indica & - & Scrophulariaceae & $\mathrm{H}$ & Cultivated land \\
\hline $\begin{array}{l}\text { Lippia alba (Mill.) N. E. Br. Ex Britt. } \\
\text { \&Wilson }\end{array}$ & Bhuiokra & Verbenaceae & $\mathrm{H}$ & Roadside \\
\hline Litchi chinensis Sonn. & Lichu & Sapindaceae & $\mathrm{T}$ & Homestead \\
\hline $\begin{array}{l}\text { Ludwigia hyssopifolia } \text { G. Don) } \\
\text { Excell apud A \&R. Fernandes }\end{array}$ & Panilong & Onagraceae & $\mathrm{H}$ & Cultivated land \\
\hline Ludwigia repens Forst. & Molsi & Onagraceae & $\mathrm{H}$ & Cultivatedland \\
\hline Luffa cylindrical (L.) M. Roem. & Dundul & Cucurbitaceae & $\mathrm{C}$ & Homestead \\
\hline Mangifera indica $\mathrm{L}$. & Aam & Anacardiaceae & $\mathrm{T}$ & Homestead \\
\hline $\begin{array}{l}\text { Mariscus squarrosus (L.) C. B. } \\
\text { Clarke }\end{array}$ & Sedge & Cyperaceae & $\mathrm{H}$ & Cultivated land \\
\hline Melia azederach $\mathrm{L}$. & Goraneem & Meliaceae & $\mathrm{T}$ & Homestead \\
\hline Merremia peltata (L.) Hallier f. & & Convolvulaceae & $\mathrm{C}$ & Roadside \\
\hline Merremia umbelata $($ L.) Hallier f. & merrimia & Convolvulaceae & $\mathrm{C}$ & Roadside \\
\hline Mikania micrantha Kunth & Assamilata & Asteraceae & $\mathrm{C}$ & Roadside \\
\hline $\begin{array}{l}\text { Momordica cochinchinensis (Lour.) } \\
\text { Spreng }\end{array}$ & Bonkakrol & Cucurbitaceae & $\mathrm{C}$ & Roadside \\
\hline Morinda citrifolia $\mathrm{L}$. & Banach & Rubiaceae & $S$ & Homestead \\
\hline Moringa oleifera Lamk. & Shajna & Moringaceae & $\mathrm{T}$ & Homestead \\
\hline $\begin{array}{l}\text { Mosla dainthera (Buch.-Ham. ex } \\
\text { Roxb.)Maxim. }\end{array}$ & - & Lamiaceae & $\mathrm{H}$ & Homestead \\
\hline Mucuna gigantea (Willd.) DC. & Bara-alkuchi & Fabaceae & $\mathrm{C}$ & Roadside \\
\hline Musa paradisiaca $\mathrm{L}$. & Kola & Musaceae & $\mathrm{H}$ & Homestead \\
\hline Nelsonia canescens (Lamk.) Spreng. & - & Acanthaceae & $\mathrm{H}$ & Roadside \\
\hline Nerium indicum Mill. & Korobi & Apocynaceae & $S$ & Roadside \\
\hline Nymphaea alba $\mathrm{L}$. & Shadashapla & Nymphaeaceae & $\mathrm{H}$ & Cultivated land \\
\hline Nymphaea nauchali Burm. & Shapla & Nymphaeaceae & $\mathrm{H}$ & Cultivated land \\
\hline Nymphaea rubra Roxb. Ex Andr. & Lalshapla & Nymphaeaceae & $\mathrm{H}$ & Cultivated land \\
\hline Nympheae pubescens Willd. & Shapla & Nymphaeaceae & $\mathrm{H}$ & Cultivated land \\
\hline Nypa fruticans Wurmb. & Goalpata & Arecaceae & $S$ & Mangrove \\
\hline Ocimum sanctum $\mathrm{L}$. & Tulsi & Lamiaceae & $\mathrm{H}$ & Homestead \\
\hline Operculina turpethum (L.) S. Manso. & - & Convolvulaceae & $\mathrm{C}$ & Roadside \\
\hline Oryza sativa $\mathrm{L}$. & Motadhan & Poaceae & $\mathrm{H}$ & Cultivated land \\
\hline Oxalis corniculata $\mathrm{L}$. & Amrul & Oxalidaceae & $\mathrm{H}$ & Roadside \\
\hline
\end{tabular}


Contd.

\begin{tabular}{|c|c|c|c|c|}
\hline Scientific name & Local name & Family & Habit & Habitat \\
\hline Paspalum distichum $\mathrm{L}$. & Gitlaghas & Poaceae & $\mathrm{H}$ & Cultivated land \\
\hline Paspalum vaginatum $\mathrm{Sw}$. & - & Poaceae & $\mathrm{H}$ & Meadow \\
\hline Pedilanthus tithymaloides Poit. & Chita & Euphorbiaceae & $\mathrm{H}$ & Homestead \\
\hline Pendanus foetida & Keakanta & Pandanaceae & $\mathrm{H}$ & Cultivated land \\
\hline Phaulopsis imbricata (Forssk.) Sweet & Kantasi & Acanthaceae & $\mathrm{H}$ & Roadside \\
\hline Phoenix paludosa Roxb. & Hetal & Arecaceae & $\mathrm{S}$ & Mangrove \\
\hline Phoenix sylvestris (L.)Roxb. & Khejur & Arecaceae & $\mathrm{T}$ & Roadside \\
\hline $\begin{array}{l}\text { Phragmites karka (Retz.) Trin.ex. } \\
\text { steud. }\end{array}$ & Nol & Poaceae & $\mathrm{H}$ & Mangrove \\
\hline Phyla nodiflora (L.) Greene & Kanghas & Verbenaceae & $\mathrm{H}$ & Cultivated land \\
\hline Phyllanthus reticulatus Poir. & Sitki & Euphorbiaceae & $\mathrm{S}$ & Roadside \\
\hline Physalis minima $\mathrm{L}$. & Potpoti & Solanaceae & $\mathrm{H}$ & Roadside \\
\hline Pithecellobium dulce (Roxb.) Benth. & Khoibabla & Mimosaceae & $\mathrm{T}$ & Homestead \\
\hline Polygonum blebeium $\mathrm{R}$. Br. & - & Polygonaceae & $\mathrm{H}$ & Cultivated land \\
\hline Polygonum flaccidum Roxb. & - & Polygonaceae & $\mathrm{H}$ & Cultivated land \\
\hline Pongamia pinnata $($ L.) Pierre & Koroj & Caesalpiniaceae & $\mathrm{T}$ & Homestead \\
\hline Porteresia coarctata (Roxb.) Tateoka & Urigass & Poaceae & $\mathrm{H}$ & Meadow \\
\hline Portulaca oleracea $\mathrm{L}$. & Nuainashak & Portulacaceae & $\mathrm{H}$ & Cultivated land \\
\hline Potamogeton pectinatus $\mathrm{L}$. & Gechu & Potamogatonaceae & $\mathrm{H}$ & Cultivated land \\
\hline Psidium guajaba $\mathrm{L}$. & Peara & Myrtaceae & $\mathrm{T}$ & Homestead \\
\hline $\begin{array}{l}\text { Psilotrichum ferrugineum (Roxb.) } \\
\text { Moq.-Tand. }\end{array}$ & Putishak & Amaranthaceae & $\mathrm{H}$ & Cultivated land \\
\hline $\begin{array}{l}\text { Psophocarpus tetragonolobus (L.) } \\
\text { DC. }\end{array}$ & Wingseem & Fabaceae & $\mathrm{C}$ & Homestead \\
\hline Punica granatum $\mathrm{L}$. & Dalim & Punicaceae & $\mathrm{S}$ & Homestead \\
\hline Raphanus sativus $\mathrm{L}$. & Mulashak & Brassicaceae & $\mathrm{H}$ & Cultivated land \\
\hline Ricinus communis $\mathrm{L}$. & Keron & Euphorbiaceae & $\mathrm{S}$ & Homestead \\
\hline Rotala indica (Willd.) Koehne & - & Lythraceae & $\mathrm{H}$ & Cultivated land \\
\hline Ruelia tuberosa $\mathrm{L}$. & Ruelia & Acanthaceae & $\mathrm{H}$ & Roadside \\
\hline Saccharum officinerum $\mathrm{L}$. & Akh & Poaceae & $\mathrm{H}$ & Cultivated land \\
\hline Saccharum spontaneum $\mathrm{L}$. & Chan & Poaceae & $\mathrm{H}$ & Mangrove \\
\hline Sapium indicum Willd. & Harua & Euphorbiaceae & $\mathrm{T}$ & Roadside \\
\hline Sarcolobus carinatus Wall. & - & Asclepiadaceae & $\mathrm{C}$ & Mangrove \\
\hline $\begin{array}{l}\text { Schumannianthus dichotomus } \\
\text { (Roxb.) Ganep. }\end{array}$ & Patipata & Meratnaceae & $\mathrm{H}$ & Homestead \\
\hline Scirpus articulatus $\mathrm{L}$. & Chesra & Cyperaceae & $\mathrm{H}$ & Cultivated land \\
\hline Scoparia dulcisL. & Chinipata & Scropulariaceae & $\mathrm{H}$ & Homestead \\
\hline Sesbania grandiflora (L.) Pers. & Bakul ful & Fabaceae & $\mathrm{S}$ & Homestead \\
\hline $\begin{array}{l}\text { Siplanthes acmella } \\
\text { (L.) Murray not (L.) L. }\end{array}$ & Spilanthes & Asteraceae & $\mathrm{H}$ & Homestead \\
\hline Solanum indicum Sensu C.B. Clark & Futki begun & Solanaceae & $\mathrm{S}$ & Homestead \\
\hline Solanum melogena $\mathrm{L}$. & Begun & Solanaceae & $\mathrm{H}$ & Homestead \\
\hline Solanum nigrum $\mathrm{L}$. & Titbegun & Solanaceae & $\mathrm{H}$ & Roadside \\
\hline Solanum virginianum $\mathrm{L}$. & Bonbegun & Solanaceae & $\mathrm{H}$ & Roadside \\
\hline Sonneratia apetala Buch.-Ham. & Keora & Sonneratiaceae & $\mathrm{T}$ & Mangrove \\
\hline
\end{tabular}


Contd.

\begin{tabular}{|c|c|c|c|c|}
\hline Scientific name & Local name & Family & Habit & Habitat \\
\hline Sonneratia caseolaris (L.)Engl. & Soilla & Sonneratiaceae & $\mathrm{T}$ & Mangrove \\
\hline Spondias pinnata (L. f.) Kurz. & Deshi amra & Anacardiaceae & $\mathrm{T}$ & Homestead \\
\hline Stephania japonica (Thunb.) Miers & Muchchanilata & Menispermaceae & $\mathrm{C}$ & Roadside \\
\hline Swietenia mahagoni (L.) Jacq. & Mehagoni & Meliaceae & $\mathrm{T}$ & Homestead \\
\hline Syzygium cumini (L.) Skeels & Kalojam & Myrtaceae & $\mathrm{T}$ & Homestead \\
\hline Syzygium fruticosum (Roxb.) DC. & Bhutijam & Myrtaceae & $\mathrm{T}$ & Homestead \\
\hline $\begin{array}{l}\text { Syzygium malaccense (L.) Merr. \& L. } \\
\text { M. Perry }\end{array}$ & Jamrul & Myrtaceae & $\mathrm{T}$ & Homestead \\
\hline $\begin{array}{l}\text { Syzygium samaracens (Blume) Merr. } \\
\text { \& Perry }\end{array}$ & Golapjam & Myrtaceae & $\mathrm{T}$ & Homestead \\
\hline Tabarnaemontana recurva Roxb. & Togor & Apocynaceae & S & Homestead \\
\hline Tagetes patula $\mathrm{L}$. & Gada & Asteraceae & $\mathrm{H}$ & Homestead \\
\hline Tamarindus indica $\mathrm{L}$. & Tentul & Caesalpiniaceae & $\mathrm{T}$ & Homestead \\
\hline Tamarix gallica $\mathrm{L}$. & Nonajau & Tamaricaceae & $\mathrm{S}$ & Mangrove \\
\hline $\begin{array}{l}\text { Terminalia arjuna (Roxb. ex DC.) } \\
\text { Wight \& Arn. }\end{array}$ & Arjun & Combretaceae & $\mathrm{T}$ & Roadside \\
\hline Terminalia bellirica (Gaertn.) Roxb. & Bohera & Combretaceae & $\mathrm{T}$ & Homestead \\
\hline Terminalia catappa $\mathrm{L}$. & Katgolap & Combretaceae & $\mathrm{T}$ & Homestead \\
\hline Terminalia chebula (Gaertn.)Retz. & Bohera & Combretaceae & $\mathrm{T}$ & Homestead \\
\hline Thespesia lampasCav.) Dalz. \& Gibs & Boloi & Malvaceae & $\mathrm{S}$ & Mangrove \\
\hline $\begin{array}{l}\text { Thespesia populnea (L.) Sol. Ex } \\
\text { Corr. }\end{array}$ & Shon boloi & Malvaceae & $\mathrm{S}$ & Mangrove \\
\hline $\begin{array}{l}\text { Tilanthera phyloxeroides (Mart.) } \\
\text { Moq. }\end{array}$ & Tilanthera & Asteraceae & $\mathrm{H}$ & Cultivated land \\
\hline $\begin{array}{l}\text { Tinospora cordifolia (Willd.) Hook.f. } \\
\& \text { Thoms. }\end{array}$ & Gulancha & Menispermaceae & $\mathrm{C}$ & Homestead \\
\hline Toona ciliate M. Roem. & Toon & Meliaceae & $\mathrm{T}$ & Roadside \\
\hline Trewia nudiflora $\mathrm{L}$. & Pidali & Euphorbiaceae & $\mathrm{T}$ & Homestead \\
\hline Typha elephantina Roxb. & Hogla & Typhaceae & $\mathrm{H}$ & Cultivated land \\
\hline Urena lobata $\mathrm{L}$. & Jogagota & Malvaceae & $\mathrm{H}$ & Homestead \\
\hline Utricularia exoleata $\mathrm{R} . \mathrm{Br}$. & Jhaji & Utriculariaceae & $\mathrm{H}$ & Cultivated land \\
\hline Vernonia cinerea $($ L.) Less. & Kuksim & Asteraceae & $\mathrm{H}$ & Roadside \\
\hline Vigna unguiculata (L.) Walp. & Borboti & Fabaceae & $\mathrm{C}$ & Homestead \\
\hline Vitex negundo $\mathrm{L}$. & Nishinda & Verbenaceae & $\mathrm{S}$ & Roadside \\
\hline Vitex trifolia L.f. & Neelnishinda & Verbenaceae & $\mathrm{S}$ & Roadside \\
\hline Wedelia calendulacea $(\mathrm{L}$.$) Less.$ & Mohabingaraj & Asteraceae & $\mathrm{H}$ & Mangrove \\
\hline Xanthium indicum Koen. ex Roxb. & Ghagrashak & Asteraceae & $\mathrm{H}$ & Cultivated land \\
\hline Xanthosoma violaceum Schott & Dudkachu & Araceae & $\mathrm{H}$ & Homestead \\
\hline Xylocarpus granatum Koen. & Dundul & Meliaceae & $\mathrm{T}$ & Mangrove \\
\hline $\begin{array}{l}\text { Xylocarpus moluccensis (Lamk.) } \\
\text { Roem. }\end{array}$ & Posur & Meliaceae & $\mathrm{T}$ & Mangrove \\
\hline Ziziphus mauritiana Lamk. & Boroi & Rhamnaceae & $\mathrm{T}$ & Homestead \\
\hline Zoysia matrella (L.) Merr. & Gass & Poaceae & $\mathrm{H}$ & Meadow \\
\hline Zoysia tenuifolia Willd. ex Thiele & Gass & Poaceae & $\mathrm{H}$ & Meadow \\
\hline
\end{tabular}

( $\mathrm{T}=$ tree, $\mathrm{S}=$ shrub, $\mathrm{H}=$ herb, $\mathrm{C}=$ climber). 
Two tayers of dams were made all around the Island to save it from hurricane and high tidal surges. The dams are criss-cross by many roads made by the local government to facilitate communication among the people living in and around the dams. Such dams and roads were planted by the forest department using a number of both native and exotic species. The noteworthy species are Samanea saman, Acacia catechu, Borassus flabelifer, Phoenix syvetris, Casuarina litoralis, Acacia auriculiformis, Acasia maengeum, Artocarpus heterophyllus, Calophylum innophylum, Eucalyptus camaldulensis, Dalbergia sissoo, Ehretia serrata, Ficus benghalensis, Sapium indicum, Toona ciliata, Gmelina arborea, Ficus racemosa, Leucaena leucocephala, Terminalia arjuna, Ficus rumphii, Excoecaria agallocha, Cassia siamea and Cassia fistula. Some bushy plants were also found in both sides of the road. The major species are Ricinus communis, Cajanus cajans, Sapium indicum, Excoecaria agallocha, Cassia alata, Calotropis procera, Calotrpis gigantean, Hibiscus pupolnea and Vitex negundo. Many climber species were also ornamented the road sides. Most common species are Mikania cordata, Caesalpinia bunduc, Canavalia ensiformis, Cuscuta reflexa, Merremia umbellate, Operculina turpethum, Stephania japonica, Anodendron paniculatum and Canavalia maritima.

Each homestead was planted by a good number of tree species. The appearance of such homestead looks like a segment of mini forest. During our survey Moringa oleifera, Acacia nilotica, Aegle marmelose, Albizia lebbeck, A. procera, A. richardiana, Samanea saman, Anacardium occidentale, Annona squamosa, Anthocephalus chinensis, Aphanamixis polystachya, Areca catechu, Artocarpus lacucha, Averrhoa carambola, Azadirachta indica, Bambusa balcooa, Bombax ceiba, Citrus maxima, Cocos nucifera, Ziziphus mauritiana, Trewia nudiflora, Terminalia chebula, Terminalia bellirica, Tamarindus indica, Syzygium malaccense, S. S. cumini, Swietenia mahagoni, Spondias pinnata, Psidium guajaba, Pongamia pinnata, Pithecellobium dulce, Melia azederach, Mangifera indica, Litchi chinensis, Lawsonia inermis, Lannea coromandelica, Erythrina indica, Diospyros malabarica and Diospyros blancoi were recorded.

Apart from the dams and homesteads, maximum land of the island is highly fertile. Local people use such land ones in a year for rain feed aman rice and fish production. During winter and summer some of the lands are used for winter crops and summer crops. Winter and summer crops are chili, watermelon, sweetpumpkin, sweetpotato, tomato and legumes. Some aquatic seasonal plants grow in rainy season. The most common plants recorded are Potamogeton pectinatus, Eichhornia crassipes, Jussiaea repen, Hydrila verticillata, Nymphaea pubescens, Nymphaea nouchali, Nymphaea rubra, Nymphaea capensis, Ipomoea aquatica, Tilanthera phyloxeroides, Alternanthera sessilis, Baccopa monnieri, Commelina benghalensis and also a good number of sedges and grasses. In summer the land was covered by a number herbaceous plant. Among them the common species are Baccopa monnieri, Dentella repens, Psilotrichum ferrugineum, Polygonum plebejum, Phyla nodiflora, Grangea madarspatana, Xanthium indicum, Portulaca oleracea, Heliotropium curassavicum, Heliotropium indicum, Eclipta prostrata and 
Alternanthera sessilis. A rare occurrence of Typha elephantina (Hogla) and Phragmitis karka (Nol) was also recorded in the wetland.

The following five species occurring in the island seem to be rare in the habitat. These are Sarcolobus carinatus, Tamarix gallica, Calophylum inophylum, Typha elephentanea and Phragmitis karka. To confirm their status further detailed survey is needed. The survey also recorded the occurrence of one species, namely Dolichandrone spathacea (Ara et al. 2013) in the Island that had already been listed as threatened in Bangladesh. A good number of medicinal plants was identified that plays an important role for the primary healthcare of local people of the island. Priority should be given for their conservation. The recorded species in the Island are Sonneratia apetala, Sonneratia caseolaris, Nipa fruticans, Centella asiatical, Mangifera indica, Scoparia dulcis, Mikania cordata, Ipomoea fistolusa, Kalanchoe pinnata, Terminalia arjuna, Stephania japonica, Cassia alata, Terminalia belliricha (Bohera), Diilenia indica (Chailta), Terminalia chebula (Horitaki), Terminalia arjuna (Arjun), Eupatorium odoratum (Pisais), Mikania scandens (Refugeelata), Cynodon dactylon (Durba), Colocasia esculenta (Kachu), and Ficus racemosa (Jogdumur).

Exotics and invasive species are a part of total floristic composition of the island. Some exotics, such as Acacia auriculiformis, Acacia mengium, Eucalyptus camaldulensis, leucaena leucocephala, and Cassia siamea were planted in the island area. Invasive species of the island are Eichhornia crassipes, Mikania cordata (Refugeelota), Chromolaena odorata (Pisais), Ipomoea fistulosa, Ageratum conyzoides, and Xanthium indicum bonplandianum. Such species are a challenge to the management of the plant diversity of the Island. A good number of wildlife supporting plant species namely by Sonneratia apetala, Sonneratia caseolaris, Avicenneia alba, Ficus benghalensis, Ficus racemosa, Ficus rhumphii, Syzygium cummuni, Sizygium fruticosum and Tamarindus indica was recorded from the island. Such species play an important role in conservation of biodiversity.

Char Kukri Mukri Island is very interesting area for eco-tourism. During this survey a number of features of the Island was indentified which has great values for conservation and ecotourism. Such features are to watch the isolated and remote Island facing to the Bay of Bengal; to watch the presence of coastal belt plantations turned into natural ecosystem; to enjoy mangrove forest; to observe the presence of introduced wildlife with their natural population; to meet friendly local people and also can enjoy local hospitality with fresh sea fish; to enjoy serine and virgin environment; to roaming and cruising all around the island by boat; to observe natural succession in the newly accreted Island; to provide huge opportunity for nature photographers; to watch shore and aquatic birds paradise. 

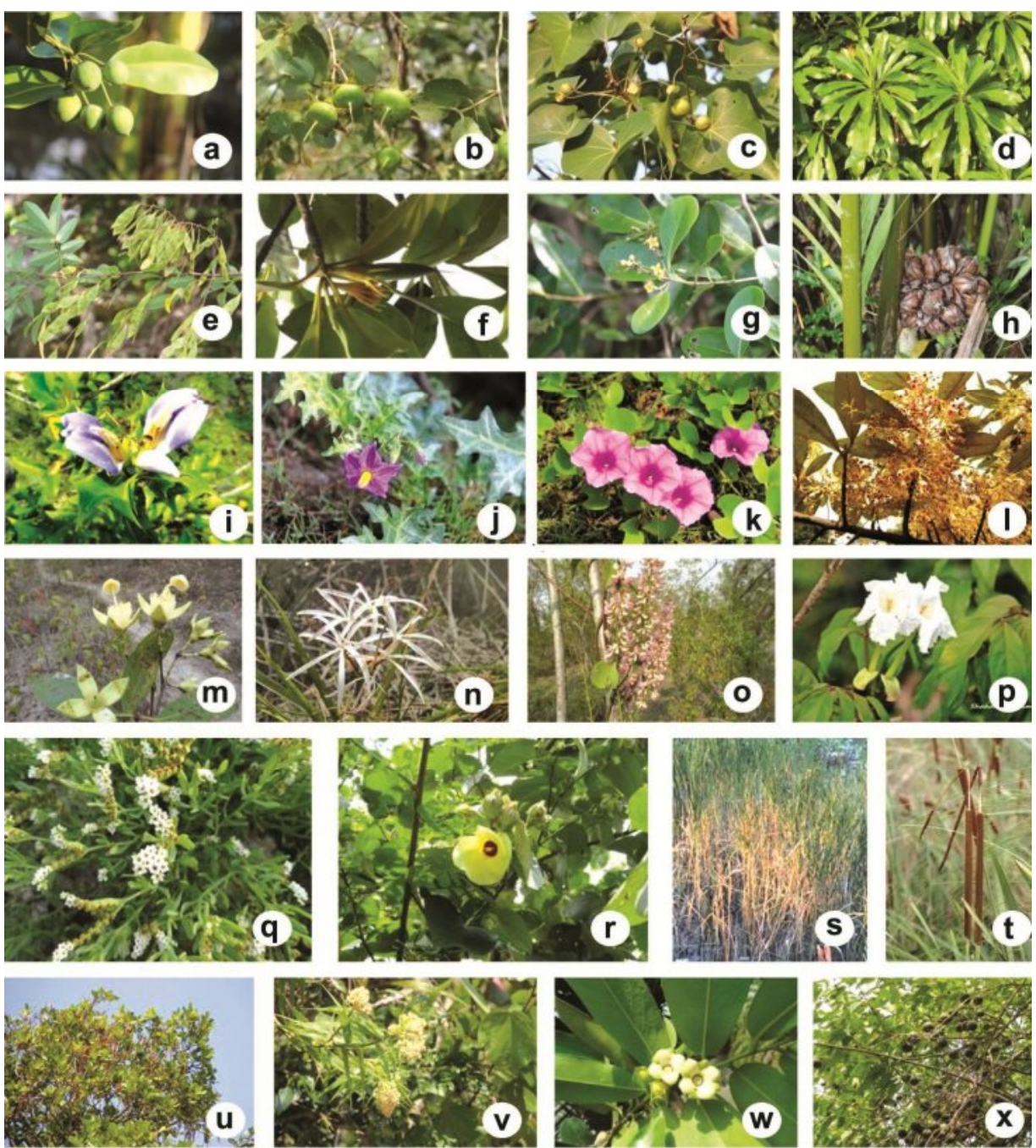

Plate 1. a. Calophyllum inophyllum, b. Sonneratia caseolaris, c. Thespesia lampas, d. Cerbera manghas, e. Derris scandens, f. Barringtonia acutangula, g. Avicennia officinalis, h. Nypa fruticans, i. Acanthus ilicifolius, j. Solanum virginianum, $k$. .Ipomoea pes-caprae, 1. Heritiera fomes, $m$. Sonneratia apetala, n. Crinum amoenum, o. Derris trifoliata, $\mathrm{p}$. Dolichandrone spathacea, q. Heliotropium curassavicum, r. Thespesia populnea, s. Porteresia coarctata, t. Typha elephantine, u. Barringtonia acutangula, v. Flagillaria indica, w. Diospyros malabarica, x. Sapium indicum.

Based on observations and discussion with local people and foresters it is evident that the island is not yet facing major threats. But the east part of the island is facing erosion 
during rainy season. The species planted there are Pongamia pinnata (Koroz), Barringtonia acutangula (Hizol), Crateva nurvala (Baorun), Trewia nudiflora (Pidali) and Acacia catechu (Babla) all of which are fresh water enduring species. Initially such species were doing better in producing branches and canopy. But their root systems are poorly developed. During high tide period the wave actions made them uprooted easily. Mangrove species like Sonneratia apetala (Keora), Sonneratia caseolaris (Soila), Avicennia officinalis (Baine) and Excoecaria agallocha (Geoa) were found to grow well in the intertidal zone. They have strong root systems and can withstand with high wave action during rainy season. Navigation to the island is one of the major constrains. Facilities and man power of local forest department are not much adequate. Introduction of exotics by forest department and BFRI are also noticeable. Grazing by buffalos in the mangrove forest area and newly accreted lands were also observed.

In order to manage the Island local knowledge based policy is very necessary. During the field trips we discussed with local forest personals, local elites and general people to find some clues for formulating recommendations. A number of suggestions which are made based on our visit experiences are: to undertake short term and long term management plans, to develop eco-tourism, to ensure security for tourist, to develop infra-structure for tourism including road construction, guest houses with local food supply, to create the sources of fresh water both for human and wildlife, to create stairs in river station to make easy movement for tourist, to establish watch towers to enjoy the beauty of the bay, to introduce more tourist boats to facilitate movement, to record local knowledge from the elders about nature and adaptation and to record health care knowledge of local people, to introduce tourist police using coast guards, to create awareness programs about environment, biodiversity and wildlife, to increase literacy rate of local people, to accelerate plantation programs using local species, to provide risk allowance for the people who involved in forest management process, to increase capacity of forest and forest personals, to develop modern infrastructures for forest personals, to detect and remove invasive species, to avoid exotics in plantation programs to arrange traditional knowledge based cultural program, to create traditional medicinal knowledge sharing programs, finally to ensure land ownership and forest territory using GIS map.

\section{Acknowledgements}

Bangladesh Forest Department and local forest office of Bhola, Char Fassion and Char Kuri Mukri are greatly acknowledged for financial support and facilities. The authors are also thankful to the wildlife rescue center of Jahangirnagar University for implementing research work.

\section{References.}

Ahmed, Z.U., Z.N.T., Begum, M.A., Hassan, M., Khondker, S.M.H., Kabir, M., Ahmad, A.T.A., Ahmed, A.K.A. Rahman and E.U. Haque (Eds) 2008a. Encyclopedia of Flora and Fauna of 
Bangladesh, Vol. 6. Angiosperms: Dicotyledons (Acanthaceae - Asteraceae). Asiatic Society of Bangladesh, Dhaka, pp. 1-408.

Ahmed, Z.U., M.A. Hassan, Z.N.T. Begum, M. Khondker, S.M.H. Kabir, M. Ahmad, A.T.A. Ahmed, A.K.A. Rahman and E.U. Haque, (Eds) 2008b. Encyclopedia of Flora and Fauna of Bangladesh, Vol. 12. Angiosperms: Monocotyledons (Orchidaceae - Zingiberaceae). Asiatic Society of Bangladesh, Dhaka, pp. 1-552.

Ahmed, Z.U., M.A. Hassan, Z.N.T. Begum, M. Khondker, S.M.H. Kabir, M. Ahmad, A.T.A. Ahmed, A.K.A. Rahman and E.U. Haque, (Eds) 2009b. Encyclopedia of Flora and Fauna of Bangladesh, Vol. 7. Angiosperms: Dicotyledons (Balsaminaceae - Euphorbiaceae). Asiatic Society of Bangladesh, Dhaka, pp. 1-546.

Ahmed, Z.U., M.A. Hassan, Z.N.T. Begum, M. Khondker, S.M.H. Kabir, M. Ahmad, A.T.A. Ahmed, A.K.A. Rahman and E.U. Haque, (Eds) 2009c. Encyclopedia of Flora and Fauna of Bangladesh, Vol. 8. Angiosperms: Dicotyledons (Fabaceae - Lythraceae). Asiatic Society of Bangladesh, Dhaka, pp. 1-478.

Ahmed, Z.U., M.A. Hassan, Z.N.T. Begum, M. Khondker, S.M.H. Kabir, M. Ahmad and A.T.A. Ahmed, (Eds) 2009d. Encyclopedia of Flora and Fauna of Bangladesh, Vol. 9. Angiosperms: Dicotyledons (Magnoliaceae - Punicaceae). Asiatic Society of Bangladesh, Dhaka, pp. 1-488.

Ahmed, Z.U., M.A. Hassan, Z.N.T. Begum, M. Khondker, S.M.H. Kabir, M. Ahmad and A.T.A. Ahmed, (Eds) 2009e. Encyclopedia of Flora and Fauna of Bangladesh, Vol. 10. Angiosperms: Dicotyledons (Ranunculaceae - Zygophyllaceae). Asiatic Society of Bangladesh, Dhaka, pp. 1-580.

Ahmed, Z.U., M. Khondker, Z.N.T. Begum, M.A. Hassan, S.M.H. Kabir, M. Ahmad, A.T.A. Ahmed and A.K.A. Rahman, (Eds) 2009a. Encyclopedia of Flora and Fauna of Bangladesh, Vol. 4. Algae: Charophyta-Rhodophyta (Achnanthaceae- Vaucheriaceae). Asiatic Society of Bangladesh, Dhaka, pp. 1-543.

Akter, A. and M.I. Zuberi. 2009. Invasive alien species in northern Bangladesh: Identification, inventory and impacts. International journal of biodiversity and conservation 1(5): 129134.

Alexiades, M.N. (ed.). 1996. Selected Guidelines for Ethno botanical Research: A Field Manual. The New York Botanical Garden, New York.

Ara, H., B. Khan and S. N. Uddin. 2013 (eds.) Red data book of vascular plants of Bangladesh, Vol 2. Bangladesh National Herbarium, Dhaka, Bangladesh. 280pp.

Balick, M. J., A. B. Anderson and M. F. da Silva. 1982. Plant taxonomy in Brazilian Amazonia: The state of systematic collection in regional herbaria. Brittonia 14: 463-477.

BBS, (Bangladesh Bureau of Statistics) 2011. Monthly Statistical Bulletin, December 2011. Statistics Division, Ministry of Planning, Government of the People's Republic of Bangladesh.

Cronquist, A. 1981. An integrated system of classification of flowering plants. Columbia University Press, New York, pp. 1262

Goldsmith, F. B. and Harrison, C. M. 1976. Description and analysis of vegetation. In: Champman, S. B. (ed.). Methods in plant ecology. Blackwell, Oxford, pp. 85-155.

Hossain, M.K. and M.K. Pasha. 2004. An account of the exotic flora of Bangladesh. Journal of forestry and environment 2: 99-115.

Hyland, B.P.M. 1972. A technique for collecting botanical specimens in rain forest. Flora Malesiana Bulletin, 26: 2038-2040.

Khan, M.S. and A.M. Huq 2001. The vascular flora of Chunati wildlife sanctuary in south Chittagong, Bangladesh.Bangladesh J. Plant.Taxon. 8(1): 47-64.

Khan, M.S., M.M. Rahmanand, M.M. Ali (eds.) 2001. Red Data Book of Vascular Plants of Bangladesh. Bangladesh National Herbarium. pp. 179. 
Khan, M.S., M.M. Rahman, A.M. Huq, M.M.K. Mia, and M. A. Hassan. 1994. Assesment of biodiversity of Teknaf game reserve in Bangladesh focusing on economically and ecologically important plants species. Bangladesh J. Plant. Taxon. 1(1): 21-33.

Rahman, M.O. and M.A. Hassan 1995. Angiospermic flora of Bhawal Narional Park, Gazipur, Bangladesh. Bangladesh J. Plant Taxon. 2(1\&2): 47-79.

Sajib, N. H., S.B. Uddin and M.K. Pasha, 2015. Angiospermic Plant Diversity Of Sandwip Island, Chittagong, Bangladesh. Asiat. Soc. Bangladesh, Sci. 41(2): 133-153.

Siddiqui, K.U., M.A., Islam, Z.U. Ahmed, Z.N.T. Begum, M.A. Hassan, M. Khondker, M.M. Rahman, S.M.H. Kabir, M. Ahmad, A.T.A. Ahmed, A.K.A. Rahman and E.U. Haque, (Eds) 2007. Encyclopedia of Flora and Fauna of Bangladesh. Vol. 11. Angiosperms: Monocotyledons (Agavaceae -Najadaceae). Asiatic Society of Bangladesh, Dhaka, pp. 1-399.

Uddin, M.Z, M.F. Alam, A.S.M. Rahman and M.A. Hassan. 2011. Plant Biodiversity of Fashiakhali Wildlife Santuary, Bangladesh. Accepted for publication in First Bangladesh Forestry Congress Proceeding 2011.

Uddin, S.B. and M.A. Rahman. 1999. Angiospermic flora of Himchari National Park, Cox's Bazar, Bangladesh. J. Plant Taxon. 6(1): 31-68.

Uddin, M.Z. and M.A. Hassan. 2010. Angiosperm diversity of Lawachara National Park (Bangladesh): a preliminary assessment. Bangladesh J. Plant Taxon.17 (1): 9-22.

Uddin, M.Z. and M.A. Hassan. 2004. Flora of Rema-Kalenga Wildlife Sanctuary. IUCN Bangladesh Country Office, Dhaka, Bangladesh, vi+120pp.

Uddin, M.Z., M. F. Alam, M. A. Rahman and M. A. Hassan. 2013. Diversity in angiosperm flora of Teknaf Wildlife Sanctuary, Bangladesh. Bangladesh J. Plant Taxon.20(2): 145-162.

Uddin, S.N., M.S. Khan, M.A. Hassan and M.K. Alam, 1998. An annotated checklist of angiospermic flora of Sitapahar at Kaptai in Bangladesh. Bangladesh J. Plant Taxon. 5(1): 1346.

Uddin, M.Z., M.G. Kibria and M.A. Hassan 2015. An assessment of angiosperm plant diversity of nijhum dweep (Island). Journal of Asiatic Society of Bangladesh Sci., 41(1): 19-32. 